Full length article

\title{
Maximising data to optimise animal disease early warning systems and risk assessment tools within Europe
}

\author{
Verity Horigan $^{\mathrm{a}, 1, *}$, Marco de Nardi ${ }^{\mathrm{c}, 1}$, Maria I. Crescio ${ }^{\mathrm{b}, 1}$, Agustin Estrada-Peña ${ }^{\mathrm{d}, 1}$, Amie Adkin ${ }^{\mathrm{a}}$, \\ Cristiana Maurella ${ }^{\mathrm{b}}$, Silvia Bertolini ${ }^{\mathrm{b}}$, Anais Léger ${ }^{\mathrm{c}}$, Giuseppe $\mathrm{Ru}^{\mathrm{b}}$, Charlotte Cook $^{\mathrm{a}}$, \\ Katharina Stark ${ }^{c}$, Robin R.L. Simons ${ }^{\mathrm{a}}$ \\ a Department of Epidemiological Sciences, Animal and Plant Health Agency, Woodham Lane, Addlestone, New Haw, Surrey KT15 3NB, UK \\ ${ }^{\mathrm{b}}$ Istituto Zooprofilattico Sperimentale del Piemonte, Liguria e Valle d'Aosta (IZSPLVA), Italy \\ ${ }^{\mathrm{c}}$ SAFOSO AG, Waldeggstrasse 1, CH3097 Liebefeld, Switzerland \\ ${ }^{\mathrm{d}}$ University of Zaragoza (UNIZAR), Calle de Pedro Cerbuna 12, 50009 Zaragoza, Spain
}

\section{A R T I C L E I N F O}

\section{Keywords:}

Animal disease

Early warning

Risk assessment

\begin{abstract}
A B S T R A C T
Timely and reliable data and information availability and sharing is essential for early warning, prevention and control of transboundary diseases. While there are a growing number of global datasets capable of providing information for use in early warning systems and risk assessment (RA) tools, there are currently time-consuming data cleansing and harmonisation activities which need to be carried out before they can be reliably used and combined. Thus, using global datasets as they stand can lead to errors in RA parameterisation and results due to inherent biases in the data, e.g. missing disease prevalence data treated as a zero may inadvertently penalise those countries which do report disease outbreaks as opposed to those countries which are affected by a pathogen but do not report outbreak data. It is therefore of great importance that data are clearly provided and easy to understand and that data providers strive for greater harmonisation of database standards.

In this paper the datasets utilised in the SPARE ('Spatial risk assessment framework for assessing exotic disease incursion and spread through Europe') project are described and discussed in terms of key criteria: accessibility, availability, completeness, consistency and quality. It is evident that most databases exist as information portals and not exclusively for RA purposes. Another striking issue from this assessment is the need for enhanced data sharing specifically with regards to data on illegal seizures, arthropod vector/wildlife abundance, intra-country livestock movement and national animal disease surveillance.

It is hoped that the outcomes of this work will promote discussion and exchange between data providers, including the development of standardised data exchange protocols. The transformation of datasets to a common format is a considerable challenge but recommendations could and should be made on the standardisation of datasets and reporting in order to achieve a unified approach across Europe.
\end{abstract}

\section{Introduction}

A co-ordinated and unified approach by European Union (EU) Member States (MS) to stamping out animal disease incursions at an early stage is crucial to preventing onward transmission. Whilst some diseases, such as African swine fever (ASF), threaten European boundaries, others are unpredictable and have appeared within the EU from unknown sources, such as, Bluetongue BTV-8 in the Netherlands (Mintiens et al., 2008) and the recent outbreak of Newcastle Disease in Belgium (DEFRA, 2018). Critical factors in managing any incursion are the ability to detect diseases at an early stage, to predict where further incursions may occur and to estimate to what extent native naïve animal populations are at risk of exposure.

The FAO-OIE-WHO (Food and Agriculture Organization; World Organisation for Animal Health; World Health Organization) jointly facilitates a Global Early Warning System (GLEWS) (FAO., OIE., WHO, 2018) for health threats and emerging risks at the human-animal-ecosystems interface. The ultimate goal of this system is to inform prevention and control measures, through the rapid detection and risk assessment of health threats and events of potential concern. Such

\footnotetext{
* Corresponding author.

E-mail address: verity.horigan@apha.gsi.gov.uk (V. Horigan).

${ }^{1}$ These authors contributed equally to this paper.
} 
preparedness is a key risk management strategy. Specifically, within a European setting, risk management is a distributed and collaborative process. Input to animal health risk management is provided by the Standing Committee of the Food Chain and Animal Health which has an advisory role and uses summary information provided by EU MSs. However, it does not typically conduct joint analysis of data sets across MSs and standardised processes for data sharing and joint analysis are lacking which may delay emergency response. Several projects have previously demonstrated the power of pooling European MS data into a centralised decision tool (Adkin et al., 2012; Simons et al., 2016). Such pooling of resources in the event of an incursion will benefit risk management decisions, which are often taken under significant time constraints, to assure a rapid translation from science into policy.

The area of European decision-support systems and spatial risk assessments (RA) has been developing rapidly in concert with increasing computing capacity and the availability of multiple spatial datasets. Several innovative European projects have shown that there is considerable added value that can be gained from spatial analysis and combination of available datasets (Simons et al., 2016; EFSA, 2014; Risksur, 2015). The collaborative European research project, SPARE, was proposed to develop a generic automated spatial RA framework for assessing incursion and spread of exotic animal pathogens in the EU, as discussed in detail in other papers in this issue (Simons et al., 2018; Bertolini et al., 2018). It also aimed to assess surveillance currently carried out by individual MSs within the EU and to investigate harmonisation and sharing of such data to assist an EU wide approach to disease incursion. The SPARE project provides an ideal platform to illustrate what data sets are required, and available, to initiate a European early warning system (EWS) and for a quantitative risk assessment (QRA) to be carried out and assessing how the available datasets fulfil the requirements of both the EWS and the QRA. In developing the SPARE framework consideration was also given to whether data could be easily extracted from original datasets and whether they could be automatically updated.

Risk assessment frameworks in the area of animal and public health generally comprise of release, exposure and consequence stages estimating the probability of a hazard being introduced through to the consequences of exposure of a susceptible population to the hazard in terms of health, welfare, economy etc. It was envisaged that the overarching model developed in SPARE would make rapid use of available data to identify pathways of potential risk for different modes of disease transmission within these RA stages. Rapid access to, and use of, available data on trade and human and animal contact networks to identify pathways of potential risk of incursion and/or spread for different modes of disease transmission would be of great benefit to a unified EU approach. The framework should be flexible and able to be applied to groups of unknown hazards, defined by their mode of transmission (e.g. vector-borne). This would help assure general preparedness and feed into EWS for decision makers on the possible animal health threats arising from, for example, changes in global trade.

Data quality and uneven reporting are key challenges to the validity of model outputs derived from the use of European and global datasets. A lack of data, or poor quality data, can limit the application of QRAs as the reliability of their outputs is conditional on the quality of the data inputs. Global datasets which provide data on pathogen prevalence and international trade and for which some form of quality assurance exists are freely available on the internet. These data form the cornerstone of the QRA in terms of inputs for the release stage and, as such, any omissions or inconsistencies in these datasets can lead to the QRA producing inaccurate outputs. In terms of the exposure and consequence stages of a QRA the spatial scale for which this can be carried out will again depend on data availability with regards to, for example, networks of animal movements and animal/vector density in the importing country. All of these data can introduce uncertainty into a QRA depending on the quality of the datasets used.

The approach taken in the SPARE project was to use case studies which covered the main routes of incursion for disease pathogens (Classical rabies, Bluetongue virus (BTV) and Classical swine fever (CSF)) (Horigan et al., 2018). Some parameters were, therefore, detailed pathogen-specific parameters, e.g. incubation period, probability of transmission of a pathogen given contact, which are not typically found in datasets but are usually obtained from experimental studies reported in the scientific literature. Such estimates were not the focus of this paper which investigates only those parameters that could be derived from large, freely available and updatable global datasets.

This paper investigated the limitations of datasets which were used throughout the QRA process or those held by individual MSs as part of national surveillance activities. Consideration was given to all data required, highlighting those data which were missing or which had large amounts of associated uncertainty. This paper summarises the experiences found throughout the SPARE project with regards to these data criteria, and highlights those areas in which improvements could be made to help optimise a European wide animal health EWS and QRA.

\section{Methods}

An assessment was made of the datasets available for use in the SPARE project according to their accessibility, availability, completeness, consistency and quality. For classification purposes, accessibility of data describes whether data actually exist and, if they do, if access is limited by cost or other restrictions. Data availability refers to the degree to which data can be instantly extracted and data completeness describes the extent to which all data that are needed are available compared to the amount that was expected to be obtained. Data consistency refers to data values in one dataset being consistent with values in another dataset (Bellet et al., 2012). Finally, data quality is here defined as the 'fitness for purpose' of the data for the specific needs. This, therefore, depends as much on the intended use as it does on the data itself. Additional requirements to optimise these datasets for use in a QRA were also identified.

A wide variety of relevant datasets were assessed such as disease presence in each country globally, and the scale of transmission in classes such as legal imports of meat, live animals, and vectors. The protocols for sharing of data between partners and certain international data providers were also investigated. Critical hurdles that may prevent data sharing and operation of the models under real emergency conditions due to technical, legal or organisational issues were also identified and recommendations on the standardisation and reporting of datasets in order to achieve a harmonised approach across the EU were made.

Datasets were scored as follows:

Data accessibility: low $=$ no data; medium $=$ access is limited or restricted by cost or to certain people; high = free access

Data availability: low = unable to download data; medium = extraction/downloading is possible but requires modifications; high = instant access with full extraction and updating capabilities

Data completeness: low = very little data required are available; medium $=a$ certain amount of data are available; high $=$ all data needed are available

Data consistency: low = data in different datasets shows much variation; medium = slight variation between data in different datasets but not too variable; high = data values in different datasets are consistent

Data quality: low=data does not satisfy requirements; medium = data are adequate but could be improved; high = data are fit for purpose

A general overview of the data flow from primary sources to international databases is shown in Fig. 1 illustrating, in particular, the exchange and feedback of data and information between the various 

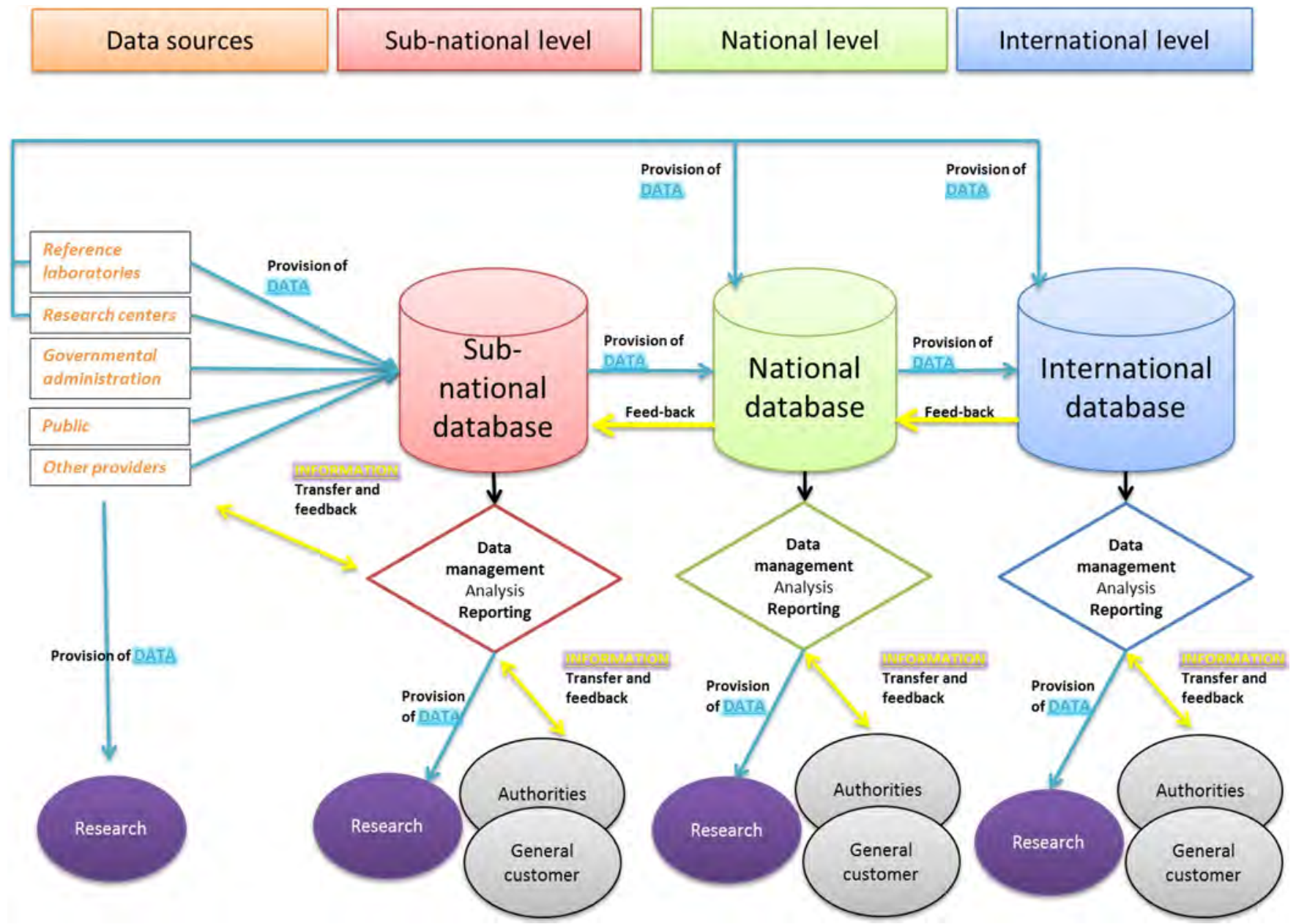

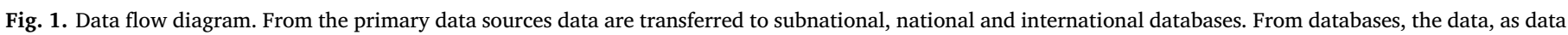

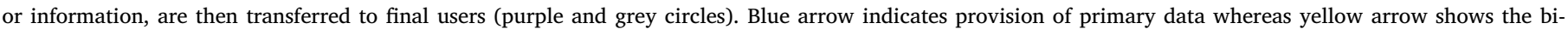

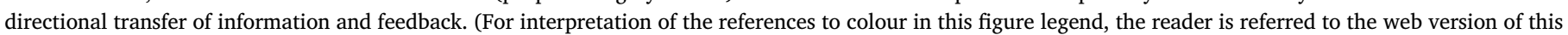
article.)

levels. It is assumed that each database, i.e. sub-national, national and international performs an internal monitoring process of data received. The data available for each stage of the QRA and the EWS are addressed in individual sections for ease of reference.

\subsection{Animal disease prevalence}

One of the main inputs needed for the SPARE QRA was the derivation of estimates for the animal disease situation in each 'origin' country of the world, requiring data on animal disease prevalence, e.g. the number of recorded outbreaks per year, number of recorded cases per outbreak and demographic livestock data such as the total number of animals per species. Table 1 lists a number of sources of information for country level pathogen prevalence data. Some datasets deal with validated data, while others include un-validated data from non-official sources. Two of the main sources that deal with validated data are the OIE World Animal Health Information System (WAHIS) (OIE 2018) and the FAO Emergency Prevention Global Animal Disease Information System (EMPRES-i) (FAO, 2018a).

\subsection{Trade of live animals and products}

There are a number of different datasets that detail trade of live animals/ products (Table 2) two of which are provided by the United Nations (UN) and one which is Europe centric and is provided by the
European Commission (EC). The commodities included in this dataset include live animals and associated meat products as well as other products that may be of interest for specific pathogens such as fruit and vegetables in terms of vector carriage.

Eurostat (2018a) is the statistical office of the EU providing statistics at European level that enable comparisons between countries and regions. Data are collected by MSs statistical authorities who verify and analyse national data and send them to Eurostat whose role is then to consolidate the data and ensure they are comparable, using harmonised methodology. Eurostat provide official European foreign trade statistics via the Comext database (Eurostat, 2018b). Intra-EU trade data are collected directly from trade operators, which send a monthly declaration to the relevant national statistical administration. Information on extra-EU and intra-EU trade is collected monthly by MSs. External trade data are subject to frequent revisions, as a consequence of errors, omissions or late declarations by information providers. As basic data consists of millions of detailed trade declarations or reports each month, it is difficult to reach complete accuracy for the published statistics. This is particularly emphasised in the intra-EU trade statistics where smallest traders are exempted from making Intrastat (the system for collecting statistics on the trade in goods between EU MSs) declarations on their monthly trade.

Other databases for trade of live animals and products include FAOstat (FAO, 2018b) and Comtrade (UN, 2018) which are both provided by the UN and therefore contain similar data. FAOSTAT provides 


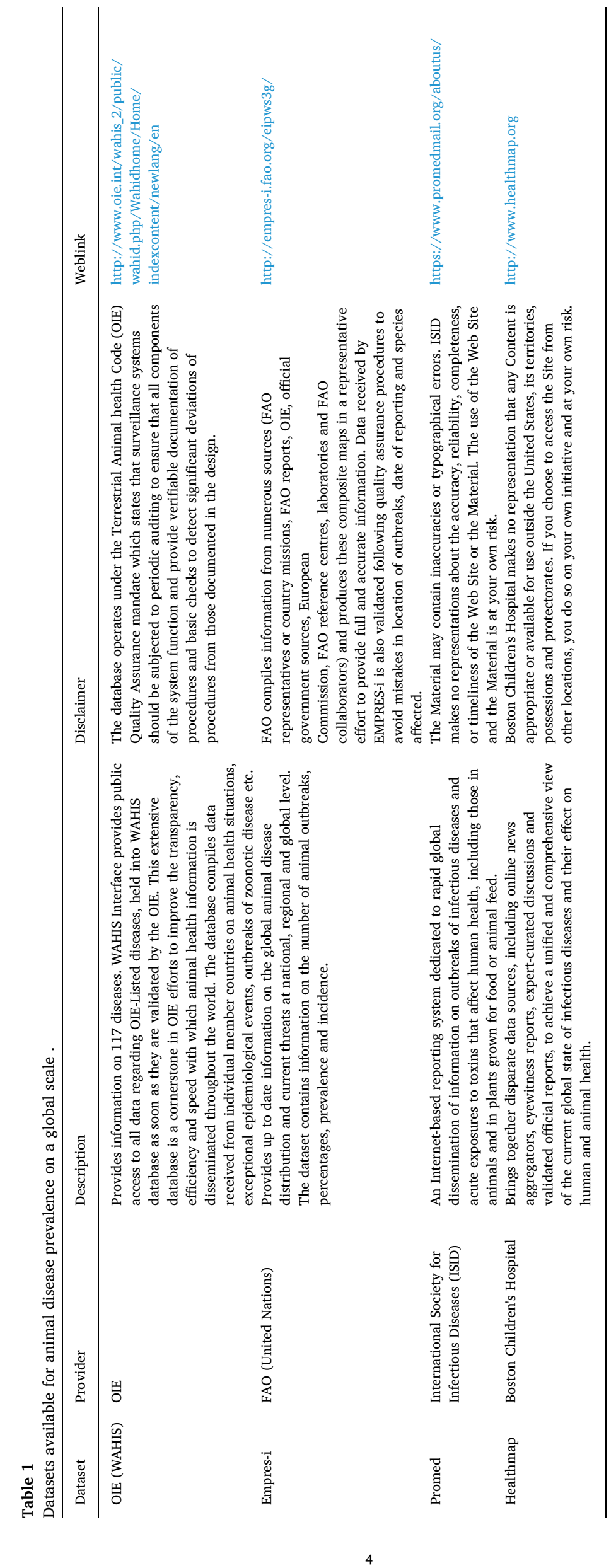


access to over 3 million time-series and cross sectional data relating to food and agriculture. FAOSTAT contains data for 200 countries and more than 200 primary products and inputs in its core data set. The Comtrade database contains detailed imports and exports statistics reported by statistical authorities of over 140 reporter countries. These data are subsequently transformed into the UN Statistics Division standard format with consistent coding and valuation. However, there are similar disclaimers as Comext in that the values of the reported detailed commodity data do not necessarily sum up to the total trade value for a given country dataset as due to confidentiality, countries may not report some of its detailed trade although this trade will be included in the total trade value. Countries do not necessarily report their trade statistics for each and every year and imports reported by one country do not coincide with exports reported by its trading partner. Differences are due to various factors including valuation, differences in inclusions/ exclusions of particular commodities, timing etc. All three databases contain details on the country of origin, the year of export, and a measure of the quantity exported (tonnes and in some cases an alternative unit, e.g. for most live animals the number of animals is given) based on commodity codes.

\subsection{Air, maritime and companion animal movement data}

Data on the movement of people by all transport modes which could also be used for movement of potential illegal items and arthropod vectors on flights, can be obtained from Eurostat (2018a), which holds data on national and international transport into EU countries. Data on the total passengers on board an aircraft and the total passengers carried arriving at a MS from a country of origin were available from the Eurostat database 'International extra-EU air passenger transport by reporting country and partner world regions and countries' (avia_paexcc) (Table 3). Air transport statistics are also available, at a cost, from the International Air Transport Association (IATA); these data are collected first hand from individual airlines rather than reporting countries.

The scale of legally internationally moved companion animals can be based on data held within the EU Trade Control Experts System (TRACES (EC, 2018)) (Table 3). These data are based on veterinary certificates for imports into the EU from third countries and include the country of origin, the importing country and also the location of the border inspection post (BIP) it went through. Data are available as a bulk download from the TRACES data warehouse. In some cases, the importing country may not be in the EU, but the BIP is; however, pets with a destination outside the EU should still be considered, as they will have stopped off in the EU to undergo inspection and thus have entered the EU.

\subsection{Livestock/Wild animal/vector distribution, abundance and movement}

The exposure of a country's native livestock population to an introduced pathogen will be dependent on the livestock demographics of individual countries. If an infected live animal enters the country a movement network analysis will be required to follow the movements of the infected animal and any potential contacts it has once it enters the country (Maurella et al., 2018; Crescio et al., 2018) This will depend on the scale of the QRA e.g. country-wide for which trade data between counties is known or individual country scale. Livestock density maps across the whole world were available for cattle, pigs, poultry, sheep, goats and buffaloes from the FAO gridded livestock of the world (FAO, 2018c) (Table 4). These maps are model predictions for the global distribution of livestock based on sub-national livestock statistics and predictor variables related to vegetation, climate, topography and demography (Robinson et al., 2014). However, such a large scale can have disadvantages, for example, when a country has different risk zones and products can only be exported from those areas which have been demonstrated to be free from disease. This can cause risk to be 

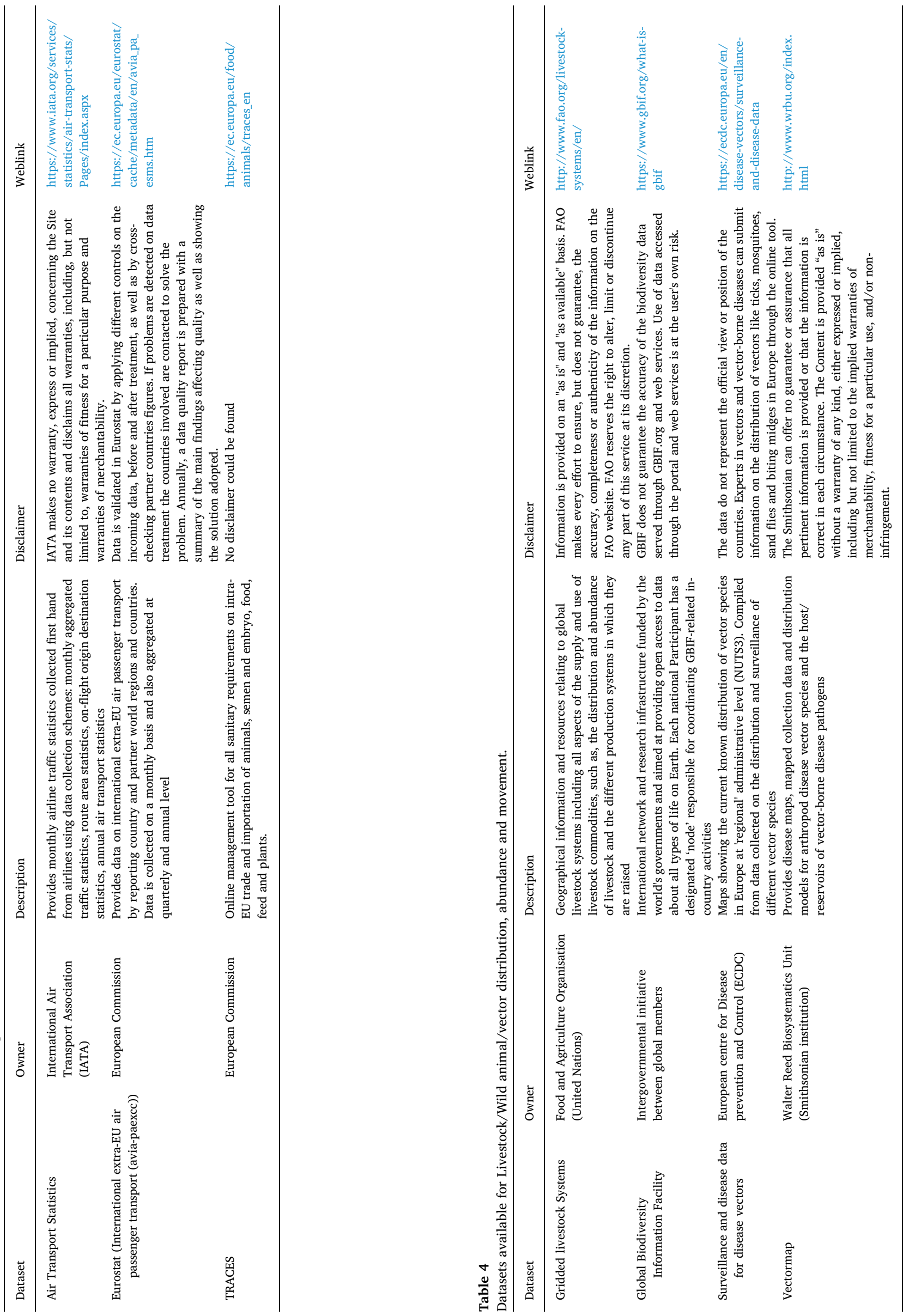


\section{incorrectly estimated.}

For a spatial QRA on a smaller scale, many EU countries have their own bespoke livestock movement datasets, detailing where and when animals are moved between holdings within a country. However, these datasets are not consistent across the EU, and, there is no centralised database where people can access them all, or even a summary of the statistics. Therefore, the exposure and consequence stages of RAs such as SPARE are limited to country level assessment.

Other less certain variables necessary to populate the data requirements of some of the pathways within QRAs included the distribution, density, abundance and movement of wild animals/birds and arthropod vectors. These populations are uncontrolled compared to livestock so official global databases for these parameters do not exist. Rather, data for these pathways are provided by predictive random sampling or proxy data such as habitat suitability. Consequently, there are no harmonised databases for wild animal density or habitat suitability maps. However, a number of researchers have attempted to produce such maps, often using different methods that don't necessarily provide the same results (e.g. see (ENETWILD, 2018) for summary of wild boar maps). Some researchers, however, have produced habitat suitability maps for multiple species that use the same methodology and thus should be consistent between species (Croft et al., 2017), including a set of habitat suitability maps developed as part of the SPARE project using the methodology from the paper in this special issue (EstradaPeña et al., 2018)-. Data that detail wild animal abundance or density are much harder to find and without some raw data on numbers density maps will suffer from high uncertainty. Some key species have had some research in this area, such as wild boar (Alexander et al., 2016), but there is no obvious official place to house such information (ENETWILD 2018; Estrada-Peña et al., 2018).

A challenge for QRA models such as SPARE when assessing arthropod vectors is data availability. Data are limited by sampling studies and are usually modelled using meteorological data and habitat suitability as proxies which introduces uncertainty within the model. This is a barrier to producing better vector borne QRAs. Species distribution models for arthropods can impact on modelling contact rates with the vertebrate hosts whilst the abundance of the population of vectors is of primary importance for modelling release and exposure, since it establishes the probable contact rates with either the reservoirs of the pathogen or the hosts.

The European Centre for Disease Prevention and Control (ECDC) (ECDC, 2018) has vector distribution maps on some species for Europe at regional administrative level or NUTS3. The NUTS classification (Nomenclature of territorial units for statistics) is a hierarchical system for dividing up the economic territory of the EU with NUTS3 being the smallest regions of which there are currently 1348. They facilitate an online tool (VectorNet (EFSA/ECDC, 2018)) by which data on distribution of vectors can be inserted directly in an online database. ECDC and the European Food Safety Authority (EFSA) collect and compile data on the distribution and surveillance of vector species to produce online maps (ECDC, 2018), providing stakeholders and general public with the most up-to-date information on vector distribution (Table 4). The data used to compile the maps are available on request. Similarly, the Walter Reed Biosystematics Unit VectorMap (Walter Reed Biosystematics Unit, 2018) facility provides disease maps, and mapped collection data and distribution models for arthropod disease vector species, including mosquitoes, ticks, sand flies, mites, and fleas, as well as the hosts/reservoirs of vector-borne disease pathogens. Collection records are searchable and downloadable so users can map and contribute their own georeferenced collection data or distribution models. These data are, however, not updateable. Other datasets such as the Global Biodiversity Information facility (GBIF) (GBIF, 2018) provide data via an international network and research infrastructure based on a core standard to make biodiversity data accessible worldwide. Information, including georeferencing records, are available for species including vectors.
The number of national studies on vector distribution/abundance from European countries has likely increased during the last decade with the threat of incursion of vector borne diseases such as BTV and Lumpy skin disease. A recent paper described a joint entomological database for large parts of Europe using surveillance and research data on the abundance of two main Culicoides species collected during the period 2007-2013 from nine individual countries (Cuéllar et al., 2018). This study suggested that future collaboration and data sharing between European countries may further improve the quality, availability and completeness of vector abundance data for use in vector borne QRAs.

\subsection{Illegal import of live animals and animal products}

Illegal import data largely suffer from the same issues as vector data, that is, lack of surveillance, use of proxy data and, perhaps most importantly, lack of data sharing between nations. Illegal carriage or trade of meat and animal by-products is known to occur, but data on the volume and frequency of such carriage/trade are limited. Individual countries hold their own seizure data but this is usually confidential. As such, no global database for illegal products exists and it is usually necessary to try to obtain the competent authority's permission to use individual databases. These data may also lack specific detail for meat products such as species of origin, normally aggregated as 'red meat' or 'white meat'.

A previous study (Simons et al., 2016) utilised data from United Kingdom (UK) customs to quantify the probability that tourists from different regions of the world would bring bushmeat into the country. These data were combined with information from Eurostat on the number of aircraft passengers from different regions of the world and an underreporting factor. Thus, an estimate for the probability that a passenger on a flight from a specific region would be carrying illegal meat in their luggage was obtained. However, this method would not account for any illegal meat introduction routes that would not appear in this dataset, such as smuggling operations that might bypass all border inspections.

\section{6. (Maurella et al., 2018; Crescio et al., 2018) national surveillance datasets}

The EU's animal health legislative framework is complex with a systematic EU approach to animal disease eradication, control and monitoring being first introduced for some diseases in 1977 (European Court of Auditors, 2016). A 2016 audit on the EU's 'Eradication, control and monitoring programmes to contain animal diseases' found that the exchange of epidemiological information between MSs and the ready access to historic results could be better supported by the relevant information systems (European Court of Auditors, 2016). Because outbreaks do not stop at national borders, information needs to be transmitted and understood regardless from where they originate. The challenges faced by institutions required to exchange data may be organizational (e.g. extent of access permitted and terms of data use, agreement between institutes about stakeholders' responsibilities etc.) or legal (e.g. compliance with national legislations and EU legislation etc.). In addition there are also technical challenges (e.g. data standardization, coding system, tools for analysis etc.) if data are to be exchanged between different databases.

The European Commission is active in promoting open data access and sharing between member states. Regarding animal health specifically, the Council Directive 82/894/EEC (EC, 1982), states that the notification of regulated animal diseases is compulsory for MSs who should notify primary outbreaks within $24 \mathrm{~h}$. The new Regulation on animal health (EU, 2016) makes clear that "system should promote optimal data availability, facilitation of data exchange, and reduction of administrative burden for the competent authorities of the MSs by merging disease notification and reporting within the Union and at 
Table 5

Summary of datasets, sources and qualitative assessment against a number of criteria.

\begin{tabular}{|c|c|c|c|c|c|c|}
\hline Data & Source & Accessibility & Availability & Completeness & Consistency & Quality \\
\hline Pathogen prevalence data & OIE & High & Medium & Medium & Medium & Medium \\
\hline Pathogen prevalence data & EMPRES-i & High & Medium & Medium & Medium & Medium \\
\hline Pathogen prevalence data & Promed & High & Medium & Medium & Medium & Medium \\
\hline Pathogen prevalence data & Healthmap & High & Medium & Medium & Medium & Medium \\
\hline Livestock demographic data & OIE & High & Medium & Medium & Medium & Medium \\
\hline Trade of Live animals/ Animal products & Comext (Eurostat) & High & High & High & Medium & High \\
\hline Trade of Live animals/ Animal products & Comtrade & High & High & High & Medium & High \\
\hline Trade of Live animals/ Animal products & Faostat & High & High & High & Medium & High \\
\hline Air and maritime human travel & Eurostat & High & High & Medium & $\mathrm{N} / \mathrm{A}$ & Medium \\
\hline Air human travel & International air transport association & Low & N/A & High & N/A & High \\
\hline Companion animal movement & Traces & Medium & Medium & High & High & High \\
\hline Wild animal density/abundance/movement & Proxy based on habitat suitability & Low & Medium & Low & N/A & Low \\
\hline Wild animal density/abundance/movement & Global Biodiversity Information Facility & Medium & Medium & Low & Low & Low \\
\hline Vector density/abundance/movement & Walter Reed Biosystematics Unit & Medium & Medium & Low & Low & Low \\
\hline Vector density/abundance/movement & European centre for Disease prevention and Control & Medium & Medium & Medium & Low & Low \\
\hline Vector density/abundance/movement & Proxy based on livestock infection & Low & Medium & Low & $\mathrm{N} / \mathrm{A}$ & Low \\
\hline Illegal import of live animals and animal products & Proxy based on papers and UK Borderforce data & Low & Medium & Low & N/A & Low \\
\hline Livestock density/movement & Food and Agriculture Organisation & High & Medium & Medium & N/A & Low \\
\hline Livestock density/movement & Individual MSs & Medium & Medium & Low & N/A & High \\
\hline National surveillance & Individual MSs & Medium & Medium & Medium & N/A & High \\
\hline
\end{tabular}

international level into one process".

The type and incidence of animal diseases vary across the EU, depending on factors which include climate, farm types, veterinary practices, and animal movements. Priority for surveillance for certain pathogens is therefore likely to vary between regions and MSs. Economic consideration is another important aspect normally used by decision makers to choose the most cost-effective solutions for risk management. As a consequence, surveillance programmes targeting the same disease can be different between European countries. For the SPARE project a survey was conducted in Italy, Switzerland and the UK to assess the early detection surveillance systems of CSF, BTV and rabies;. the evaluation was based on experts' opinion reached through a knowledge elicitation as discussed in detail in another paper in this issue (De Nardi et al., 2018).

\section{Results and discussion}

An overview of the available databases and an assessment of these data in terms of accessibility, availability, completeness, consistency and quality are shown in Table 5.

\subsection{Data accessibility}

There was a number of datasets that could not be accessed as they were 'closed' in the sense that they required passwords to access, a financial contribution or were only available on request. For example, the TRACES database, while free of charge, required a login to be provided by the EU MS competent authorities, who are not always guaranteed to provide access. Other more detailed datasets also exist for air passenger travel such as the International Air Transport Association (IATA), however, this required payment to gain access to the database and was therefore excluded from further consideration.

Another consideration when utilising data from large repositories that collate information from a large number of different sources, e.g. the Global Biodiversity Information Facility (GBIF) (GBIF, 2018), is the issue of 'sub-referencing'. For many of these sources it is necessary to reference every dataset that has contributed to the particular subset of data used. The concern about accidentally not properly referencing everything, and thus risking negative consequences, led to the consequence of not using some datasets that would otherwise have been of use. Intellectual property is clearly important if a company has spent a great deal of time, money and effort in putting together a comprehensive dataset. However, there is a counter argument to be made that these 'closed' datasets will restrict the number of users and in the long term provide less benefit to the company and society in general. More needs to be done in this area to incentivise companies to make their data freely accessible, in order to allow all developers access to the best quality data to develop more robust models.

\subsection{Data availability}

The original aim of the SPARE risk assessment was to develop methods that would allow the model to search online databases and automatically update with new data if available. Unfortunately, while some databases, such as Eurostat, had very efficient systems by which to obtain data, such as an official ' $R$ ' package (Eurostat, 2018c) that allowed data to be downloaded automatically, others did not. To obtain the OIE WAHIS data required accessing the data via multiple different search tools on the WAHIS website. Automatic availability to other databases could not be obtained for a variety of other reasons such as data being hidden behind complicated systems such as flash player (Empres-i).

The Comext database scored highly for ease of availability either via the bulk download facility (Eurostat, 2018d) or an official package in $\mathrm{R}$ to download automatically (Eurostat, 2018a). The UN Faostat and Comtrade databases also had Application programming interfaces to allow extraction of data (FAO, 2013; Comtrade, 2018).

An issue found with the Eurostat database used for air passenger data was that it is possible this figure might include EU originating passengers who board the plane between the exporting country and the final destination EU MS i.e. they are already in the EU but the data counts them as coming from the exporting country. This may result in an overestimation of passengers from the export country, but as the number of these passengers could not be identified they could not be excluded from the dataset. Some files were also marked as confidential so it is possible that not all data were fully extracted.

\subsection{Data completeness}

Both the OIE and Empres-I databases suffer from incomplete data. Missing data occurs when disease is only reported as presence without a numerical estimate of scale or no disease information is reported at all. Absence of reported disease could be considered to imply disease free status of a country, when in reality this may not be the case. Global datasets are inherently subject to differences in the quality of reporting from individual countries and the monitoring systems for disease on 
which these reports are based, including instances where cases of disease are reported but there are no reported outbreaks. This is further compounded by differences in the performance of the surveillance strategies according to the targeted livestock production system, with nomadic and pastoral systems being more difficult to survey. Other reasons for underreporting or inaccurate reports of diseases may include: poorly resourced veterinary services to detect disease presence, unwillingness to report outbreaks due to political reasons; regional differences in disease prioritisation and inadequate compensation for culled animals (Keusch et al., 2009).

Trade data, as decribed in Table 2, were available on a global and EU level and considered to be of high quality and completeness. Animal contact networks, however, whilst considered of high quality were not available at a spatial level below country level which was a major drawback within the SPARE model. It was found that data at a refined spatial level to the degree where area probability could be calculated was only available on a national basis and no sharing of data appeared to exist. In addition, there were also technical challenges, such as coordinating the use of different country coding systems, if data were to be exchanged between different databases. At the present time, this is a major barrier to carrying out spatial RA to a regional, or lower, level.

Other datasets with the lowest level of completeness were those of vectors and wildlife abundance and movement. It is likely that RAs considering these routes of entry and spread will become more widely used in the future as areas in which arthropods exhibit "vector competence" (the ability to acquire, maintain and transmit an infectious agent) expand and wildlife become increasingly important routes of pathogen entry such as wild boar and the threat of ASF in Europe.

There is a lack of adequate knowledge regarding the distribution of arthropods vectors of pathogens. Available datasets include the most important vectors of pathogens using administrative divisions (NUTS3) rather than providing reliable coordinates. While these efforts are an improvement over the data available $\sim 5$ years ago, current modelling techniques ideally need geo-referenced records. The researchers of SPARE have produced a reliable dataset of records of ticks in Europe (Estrada-Peña et al., 2018)-, but continental analysis of the distribution of most arthropod vectors of pathogens is still lacking. Researchers should be encouraged to publish records with coordinates and this information should be available in a global dataset making the known distribution of arthropod vectors of pathogens available for further studies.

Although pathogen specific parameters are not specifically considered here they are extremely important in times of an outbreak especially if the pathogen is exotic or a novel strain of an endemic pathogen. Practices and procedures that promote rapid, timely and efficient access and sharing of relevant data (i.e. diagnostic and epidemiological data related to outbreaks of diseases with intra-community trade impact) should therefore also be identified for this data.

\subsection{Data consistency}

Consistency of data between different databases was identified as an issue. Large bodies such as Eurostat, OIE and the World Bank ensure intra-consistency of databases they hold, but unfortunately there is little inter-consistency between these organisations. Creating the necessary fields in order to link the databases was time consuming and, in some instances, not possible. For example, country names were often recorded in different ways, sometimes with the full country names, or with a number of different codes. An officially recognised consistent way of reporting fields, such as using the ISO3 country code, would be of use in this instance. While this problem can be overcome with a reasonable amount of cleaning of datasets, provinces of countries are much harder to deal with. Globally, there does not appear to be a recognised standard coding system for provinces or even definition of what a province is. The EU does have the NUTS coding system (EU, 2003) for this purpose but even this has considerable heterogeneity in the size of the areas between MSs. The OIE reports case data at a province level and an attempt was made to use these data, as it is important when assessing the probability of entry of a pathogen to Europe to know, for example, if a case of ASF in Russia occurred in Kaliningrad, an exclave which borders Poland and Lithuania, or Primorsky Krai which borders China and North Korea. However, it proved too complicated to link up the province names for all countries of the world with other datasets for spatial analysis due to a combination of different spellings of province names and different definitions of some country provinces between databases.

Different trade databases sometimes gave different values for what appeared to be the same product. As stated in Table 2, this could be because, due to confidentiality, countries may not report some of its detailed trade, or report their trade statistics for each and every year or use the most recent commodity classification. Imports reported by one country did not always tally with exports reported by the trading partners for reasons stated in Table 2. However, all three trade databases scored medium for 'data consistency' i.e. slight variation between data in different datasets but not too variable, so for an EU QRA like SPARE UN data could be used to supplement data gaps within the Comext data.

National animal health surveillance systems were also assessed for inter-country consistency of data using the three cases studies: Classical rabies, BTV and CSF. The implementation of a passive surveillance for CSF in domestic and wild pigs was a shared component between all three countries assessed. Wild and domestic animals were targeted by the surveillance systems but the source of data collection differed, for example, slaughterhouses, farms or wild environments. Rabies passive surveillance of wildlife was a common component but no BTV surveillance component was common among all three countries. The study highlighted differences between the countries in the organisation of the surveillance systems even with the same objective e.g. early detection, and was related to specific contexts e.g. political dynamics, interest and specific concern of countries. Such differences between countries among Europe highlight the fact that, even under the same legislation, countries have flexibility in implementing surveillance systems (De Nardi et al., 2018) .Overall there was a lack of harmonisation of systems in Europe and consequent potential difference in terms of performance within a common trade area.

\subsection{Data quality}

The definition of data quality is the 'fitness for purpose' of the data for the originally intended specific use and it is acknowledged that these databases are not designed specifically for the data to be used in risk assessments. The EC mentions in its disclaimer that 'the data presented in Comext is of a general nature only and is not intended to address the specific circumstances of any particular individual or entity'. However, as outlined in this paper, these datasets are of particular use for QRAs and in many cases there are some improvements that could improve their value and accessibility, for a much wider audience, not just risk assessors for whom the consistency and quality of data collection in a format that facilitates analysis is vital.

To help improve data quality, it is important for data providers to have the resource for long term maintenance of the databases, including pro-active elicitation of feedback from end users and the ability to enact useful suggestions to improve functionality. Many providers, for example, Eurostat, invite feedback on the data they provide although it is currently not known what the outcome of such feedback is. It is also important for end users to provide feedback to the data providers even when un-elicited. Equally the risk assessor has a responsibility to check the quality of data available before use within a QRA. There is a concern that, despite highlighting assumptions, uncertainties and data gaps, once a model is freely available some model users may not fully appreciate the implications of areas of poor data quality or uncertainty associated with the data feeding into the model. Ideally, for each of 
these databases, the data quality should be monitored by the risk assessor before the QRA is conducted. For the SPARE model, this led to the use of an algorithm for the OIE data to compensate for missing data (Simons et al., 2017).

\section{Conclusion}

This paper describes the freely available global datasets utilised in the SPARE project and discusses some of the common issues encountered with using such datasets for risk assessments, a purpose for which they were not originally designed. A qualitative assessment was made of some specific datasets against key criteria: accessibility, availability, completeness, consistency and quality. Whilst global data of high quality were available for pathogen prevalence and trade of animals and animal products both the quality and availability of data concerning illegal trade, vector and wild animal abundance and movement was considerably lower in standard. In terms of completeness, lack of a centralised database on livestock density and movements at a regional level meant that this data requirement scored low whilst there were inconsistencies with some of the trade datasets in terms of country names and use of different commodity codes.

While there is a growing number of available global datasets capable of providing information of use for QRAs, currently there are assumptions which need to be made regarding the data before they can be used. Using global datasets as they stand can lead to errors in risk assessment parameterisation and results due to inherent biases in the data, e.g. missing data treated as a zero may inadvertently penalise those countries which do report disease outbreaks as opposed to those countries which are affected by a pathogen but do not report outbreak data (Simons et al., 2017). It is therefore of great importance that data are clearly provided and easy to understand. Consistency of data between different databases was also found to be an issue; at best it can be time consuming to create the necessary fields in order to link the databases and at worst it may not be possible. An officially recognised consistent way of reporting fields, such as using the ISO3 country code, would be of great use if taken forward by a globally recognised body such as the WHO or World Bank.

Data existed for most stages of the QRA developed in SPARE but they vary in standard for all the categories they were assessed on. It is evident that these databases exist as information portals and not specifically for use in a risk assessment setting. If data quality is defined as the 'fitness for purpose' of the data for a specific use it is not surprising that the data available does not suit all purposes. There is therefore a responsibility for the user of the data to check the data thoroughly before use. Similarly there are many areas in which the global datasets can be improved such as consistency of country names or free access to all users.

One striking issue to come out of this assessment is that of the need for data sharing specifically with regards to data on illegal seizures, vector abundance and livestock movement. Countries are obliged to report illegal seizure data to the EU so it would be of interest to see what data is actually held centrally and how this data could be applied to QRAs although it is likely that such data would be restricted by confidentially issues. If spatial QRAs are to be done at a regional-level then future sharing of such datasets is a necessary step forward to combat many of the availability and completeness data issues identified here.

Timely and reliable disease information enhances early warning and response to transboundary and high impact animal diseases and supports prevention, improved management and progressive approach to control. An assessment was, therefore, also made of national surveillance datasets in times of outbreak. Overall there was a lack of harmonisation of systems in Europe which could result in differences in proficiency of disease surveillance within a common trade area. With regards to surveillance data sharing between European countries, an objective would be to promote interaction and exchange between different providers of data to facilitate interfacing between data collection and to promote harmonised good practice for quality assurance.

The SPARE project has successfully built a model to provide a European wide EWS and QRA package to predict and manage exotic disease incursions. However, at present the data available to fulfil the model are inadequate. There remains a requirement for harmonisation of data fields and quality standards by data providers so that datasets can be utilised within one platform. This is true of arthropod vector and wild animal data, and datasets detailing individual country surveillance schemes by pathogens and control measures when an outbreak occurs.

It is hoped that the outcomes of SPARE will promote discussion and exchange between data providers, including the development of standardised data exchange protocols, ultimately encouraging an integrated EWS. The development of such a framework and transformation of datasets to a common format is a considerable challenge but recommendations could, and should, be made on the standardisation of datasets and reporting in order to achieve a unified approach across Europe. A framework for the communication of results in an emergency situation to assure a rapid translation from science into policy would be beneficial. As a potential next step towards such harmonization, the SPARE project has developed a Data interface protocol which describes the key technical, legal and organizational elements that need to be explicit and agreed between all partners (SPARE, 2018; Estrada-Peña et al., 2018).

In light of this a 'wish list' was devised by the SPARE project members to highlight those aspects of the data used in the project which could be improved for the benefit of future users:

- Standardisation of nomenclature between databases, e.g. use of ISO3 codes for countries; Latin names for animal species.

- The interface of the databases should be developed to be as userfriendly as possible, e.g. use of English language to increase accessibility for international users, and data easily retrieved; access to underlying database; advanced search functionality; ability to bulk download and an official API to facilitate automatic downloading.

- Metadata should always be available with clear description of data features. Contact information for the organization managing the database should be clearly indicated and dates of historical and future updates should be available. Wherever possible spatial data should be compiled according to geo-coordinates resolution for modelling purposes, e.g. datasets for distribution of arthropod vectors or locations of disease outbreaks.

- The access to databases should be facilitated by simplifying the registration process (in cases where registration is necessary) and request for permission process (in cases where official approval is required).

- Development of a platform for sustained, reliable and rapid exchange of data and associated metadata between EU MSs, for the purpose of trans-boundary disease management.

\section{Acknowledgements}

Funding: This work had funding agreed through the Animal Health and Welfare ERA-NET consortium (https://www.anihwa.eu/) under SPARE ('Spatial risk assessment framework for assessing exotic disease incursion and spread through Europe'). Funders are acknowledged as the Department for the Environment, Food and Rural Affairs (Defra) UK, Ministry of Health - Italy, Spanish National Institute of Agriculture and Food Research and Technology - Spain, and Federal Food Safety and Veterinary Office (FSVO) - Switzerland.

\section{References}

Adkin, A., Simmons, R., Arnold, M., 2012. Model For Evaluation of Different Options For the Monitoring of Transmissible Spongiform Encephalopathies in Cattle in the European Union (C-TSEMM) 349(55) EFSA Supporting Publications. 
Alexander, N., Massei, G., Wint, W., 2016. The European distribution of Sus Scrofa. Model Outputs from the project described within the poster - Where are all the boars? An attempt to gain a continental perspective. Open Health Data 4 (1), 1.

European Court of Auditors., Eradication, Control and Monitoring Programmes to Contain Animal Diseases. 2016.

Bellet, C., Humblet, M.-F., Swanenburg, M., Dhé, J., Vandeputte, S., Thébault, A., Gauchard, F., Hendrikx, P., De Vos, C., De Koeijer, A., Saegerman, C., Sanaa, M., 2012. Specification of Data Collection on Animal Diseases to Increase the Preparedness of the AHAW Panel to Answer Future Mandates - CFP/EFSA/AHAW/ 2010/01 354. EFSA Supporting Publications, pp. 215.

Bertolini, S., Robin, R.L., Simons, R.L., Horigan, V., Crescio, M.I., Maurella, C. Mastrantonio, G., De Nardi, M., Ru, G., Ingravalle, F., Estrada-Peña, A., Cook, C., Adkin, A., 2018. A European spatial risk assessment to detect hot spots of incursion and spread of animal exotic diseases. Microb. Risk Anal This issue.

Comtrade., The UN Comtrade Data Extraction API. 2018.

Crescio, M.I., Mastrantonio, G., Bertolini, S., Maurella, C., Adkin, A., Ingravalle, F., Simons, R., DeNardi, M., Stark, K., Estrada-Pe na, A., Ru, G., 2018. Using network analysis to identify seasonal patterns and key nodes for risk-based surveillance of pig diseases. Microb. Risk Anal This issue.

Croft, S., Chauvenet, A.L.M., Smith, G.C., 2017. A systematic approach to estimate the distribution and total abundance of British mammals. PLoS One 12 (6), e0176339.

Cuéllar, A.C., Kjær, L.J., Kirkeby, C., Skovgard, H., Nielsen, Sø A., Stockmarr, A., Andersson, G., Lindstrom, A., Chirico, J., Lühken, R., Steinke, S., Kiel, E., Gethmann, J., Conraths, F.J., Larska, M., Hamnes, I., Sviland, S., Hopp, P., Brugger, K., Rubel, F., Balenghien, T., Garros, C., Rakotoarivony, I., Allène, X., Lhoir, J., Chavernac, D., Delécolle, J.C., Mathieu, B., Delécolle, D., Setier-Rio, M.L., Venail, R., Scheid, B. Chueca, M.Á M., Barceló, C., Lucientes, J., Estrada, R., Mathis, A., Tack, W., Bødker, R., 2018. Spatial and temporal variation in the abundance of Culicoides biting midges (Diptera: ceratopogonidae) in nine European countries. Parasites Vectors 11 (1).

De Nardi, M., Léger, A., Adkin, A., Ru, G., Estrada-Peña, A., Stärk, K.D.C., 2018. Description of surveillance components related to classical swine fever, blue tongue and rabies in selected European countries: an experts knowledge elicitation. Microb. Risk Anal This issue.

DEFRA, 2018. Advice Services - International Disease Monitoring.

EC, 1982. .

EC, 2018 .

ECDC, 2018. .

EFSA, 2014. EFSA Supporting Publications, pp. 1-110.

\section{EFSA/ECDC, VectorNet. 2018.}

ENETWILD, 2018. EFSA supporting publication.

Estrada-Peña, A., Adkin, A., Bertolini, S., Cook, C., Crescio, M.I., Grosbois, V., Horigan, V., Ip, S., Leger, A., Mastrantonio, G., Maurella, C., de Nardi, M., Ru, G., Simons, R., Snary, E., Staerk, K., Taylor, R., Smith, G.C., 2018. Evaluating a mixed abiotic-biotic model for evaluating the distribution and host contact rates of an arthropod vector of pathogens: an example with Ixodes ricinus (Ixodidae). Microb. Risk Anal This issue.
EU, 2003. .

EU, 2016. .

Eurostat, 2018.

Eurostat, 2018.

Eurostat, 2018.

Eurostat, 2018. .

FAO, Accessing Data. 2013.

FAO, 2018. .

FAO, 2018. .

FAO, 2018. .

FAO., OIE., WHO, 2018.

GBIF, 2018.

Horigan, V., De Nardi, M., Simons, R.R.L., Bertolini, S., Crescio, M.I., Estrada-Peña, A., Léger, A., Maurella, C., Ru, G., Schuppers, M., Stärk, K.D.C., Adkin, A., 2018. Using multi-criteria risk ranking methodology to select case studies for a generic risk assessment framework for exotic disease incursion and spread through Europe. Prev. Vet. Med. 153, 47-55.

Keusch, G.T., Pappaioanou, M., Gonzalez, M.C., Scott, K.A., Tsai, P., 2009. Sustaining Global Surveillance and Response to Emerging Zoonotic Diseases. National Academies Press (US), Washington (DC).

Maurella, C., Mastrantonio, G., Bertolini, S., Crescio, M.I., Ingravalle, F., Adkin, A., Simons, R., De Nardi, M., Estrada -Peña, A., Horigan, V., Ru, G., 2018. Integration of network analysis into risk assessment: an animal health example. Microb. Risk Anal This issue.

Mintiens, K., Méroc, E., Mellor, P.S., Staubach, C., Gerbier, G., Elbers, A.R.W., Hendrickx, G., De Clercq, K., 2008. Possible routes of introduction of bluetongue virus serotype 8 into the epicentre of the 2006 epidemic in north-western Europe. Prev. Vet. Med. 87 (1-2), 131-144.

OIE, 2018. .

Risksur, 2015.

Robinson, T.P., William Wint, G.R., Conchedda, G., Van Boeckel, T.P., Ercoli, V., Palamara, E., Cinardi, G., D'Aietti, L., Hay, S.I., Gilbert, M., 2014. Mapping the global distribution of livestock. PLoS One 9 (5), e96084.

Simons, R., Horigan, V., De Nardi, M., Ru, G., Pena, A.E.A., Adkin, A., 2017. Mighty models from litle data grow: estimating animal disease prevalence. SVPM.

Simons, R.R.L., Horigan, V., Ip, S., Taylor, R.A., Crescio, M.I., Maurella, C., Mastrantonio, G., Bertolini, S., Ru, G., Cook, C., Adkin, A., 2018. A spatial risk assessment model framework for incursion of exotic animal disease into the European Union Member States. Microb. Risk Anal This issue.

Simons, R.R.L., Horigan, V., Gale, P., Kosmider, R.D., Breed, A.C., Snary, E.L., 2016. A generic quantitative risk assessment framework for the entry of bat-borne zoonotic viruses into the European Union. PLoS One 11 (10), e0165383.

SPARE, 2018.

UN, 2018. .

Walter Reed Biosystematics Unit, 2018. 